Mareike Schonhoff ${ }^{\star}$, Astrid Heinze, Juliette Carrillo, Annika Lopinski, Damian Großkreutz and Stefan Hanusek

\title{
Development and manufacturing of a custom made implant regarding the new European Medical Device Regulation
}

https://doi.org/10.1515/cdbme-2019-0064

\begin{abstract}
New technologies are great opportunities for personalized medicine. Custom made implants can be very helpful for patients with severe bone defects or in case of bone tumor. Through the European Union it is regulated how many possibilities and restrictions all medical devices have. Because of critical vulnerabilities a new European Medical Device Regulation (MDR) was published in May 2017 and it will enter into force in May 2020. For the manufacturers of customized products it will change the documentation of the manufacturing and tracking of serious incidents.
\end{abstract}

Patients with a pelvis defect of Paprosky IIb and higher can benefit from a custom made pelvis implant, because all planning steps according to biomechanic and bone contact to the implant can be designed and proofed during a reconstruction process. With regular modular implant systems, it probably can be a trial and error procedure during the surgery according to biomechanic and a stable position of the implant.

Based on the 3D-Reconstruction of CT-Scans of a patient with a Paprosky $2 \mathrm{~b}$ pelvis defect, a personalized acetabulum implant was designed. To maintain as much bone as possible, the implant was shaped to the remaining pelvic bone stock. Additive manufacturing gives the opportunity to produce custom made single items even in a quality that ful-fills the requirements of the MDR. Modern Selective Laser Melting (SLM) and Electron Beam melting (EBM) Systems are able to produce Titanium or $\mathrm{CoCr}$ parts in the ISO standards for Implants (ISO $5832 \mathrm{ff}$ ).

In this study the process chain, starting from the reconstruction, to the design and the production of a custom made acetabulum cup was run through on an exemplary CT-

\footnotetext{
*Corresponding author Mareike Schonhoff: Frankfurt University of Applied Sciences, Nibelungenplatz 1, Frankfurt, Germany, e-mail: schonhof@stud.fra-uas.de

Astrid Heinze, Juliette Carrillo, Annika Lopinski, Damian

Großkreutz, Stefan Hanusek: Frankfurt University of Applied

Sciences, Frankfurt, Germany
}

Data of one patient. With this example, it was shown that it is possible, to establish a process, that is able to address surgical needs for patients that benefit from those techniques.

\section{Einführung}

In Deutschland wurden 2017 rund 157.000 Hüftendoprothesen eingesetzt [1]. Aufgrund des Erreichens des hohen Lebensalters und den Wunsch auf erhaltene Mobilität, steigt diese Zahl von Jahr zu Jahr. Bei Revisionsendoprothesen kommt es wegen der individuell vorhandenen Knochensubstanz in manchen Fällen zu Schwierigkeiten bei dem Einsetzen von einer standardisierten Endoprothese. Dabei gibt es verschiedene Möglichkeiten, um trotz der Knochendefekte, die Acetabulum Pfanne im Hüftknochen fest $\mathrm{zu}$ verankern. Bei einem Paprosky Defekt $2 \mathrm{~B}$ oder höher kommt für den Patienten ein individuell hergestelltes Implantat in Frage [2]. Aufgrund der seit 2017 veröffentlichten neuen Medizinprodukteverordnung (MDR) verändert sich die Gesetzeslage in der europäischen Union für alle Medizinprodukte [3]. Somit auch für die individualisierte Endoprothetik. Mithilfe der MDR soll ein Überblick über die grundlegenden Veränderungen für die Hersteller von Medizinprodukte geschaffen und ein Prozess erarbeitet werden, der zeigt, wie eine individualisierte Hüftpfanne hergestellt werden kann.

Die neuen Technologien, die unter den Begriff des Rapid Prototyping fallen, sind besonders für die personalisierte Medizintechnik geeignet. Eine schnelle Herstellung von komplexen Sonderanfertigungen ist dadurch keine Schwierigkeit mehr. 


\section{Material und Methode}

\subsection{Medizinprodukteverordnung 2017/745/EG}

Die Medizinprodukteverordnung 2017/745/EG, welche vollständig bis zum Mai 2020 umgesetzt werden muss, wird mit der in Deutschland vorherigen Medizinprodukterichtlinie 93/42/EWG (MDD) verglichen und Unterschiede festgehalten. [3, 4]

In Bezug auf die individualisierte Endoprothetik wird ein spezieller Blickpunkt auf die MDR gelegt, um für Hersteller dieses gesonderten Bereiches einen Überblick zu geben. Die Sonderanfertigungen werden in dem Anhang XII der MDR separat behandelt.

\section{2 (Re-)Konstruktion}

Für die (Re-)Konstruktion einer individualisierten Acetabulum-Pfanne wird ein CT-Scan Datensatz einer weiblichen Patientin mittleren Alters genutzt. Diese Bilder zeigen, dass die linke Hüfte bereits einer Revision unterzogen wurde. Jedoch stimmt das Rotationszentrum der Pfanne nicht mit dem Natürlichen überein. Eine aseptische Lockerung der Hüftpfanne ist die Folge. Aufgrund dessen ist es notwendig einen erneuten Austausch des Implantates durchzuführen. Durch den nicht optimalen Sitz der vorherigen Pfanne, sind untypische Defekte entstanden, wodurch ein individuelles Implantat als Behandlungsstrategie geeignet ist.

Die Rekonstruktion aus den CT-Daten und die darauf folgende Konstruktion der Endoprothese wurden in mehreren Schritten durchgeführt (Figure 1). Zunächst wird mithilfe von Mimics (Materialise, Leuven, Belgium) der Knochen von dem Weichgewebe separiert. Zusätzlich dazu werden der Femur und das Acetabulum als getrennter Part definiert, sodass anschließend lediglich der Hüftknochen genutzt werden kann um ein Negativ für die Geometrie des Pfannenimplantates zu bilden. Mithilfe von booleschen Körpern und dem Anpassen an den virtuellen Knochen wird in Meshmixer (Autodesk, San Rafael, USA) die künstliche Hüftpfanne erzeugt. Um die stabile Verankerung mit dem Knochenzement zu realisieren, wird die Oberflächenrauheit durch Erzeugen unterschiedlicher Formstrukturen erhöht. Als letzten Schritt werden Bohrungen und ein Durchgangsloch für einen zentraler Zapfen platziert,
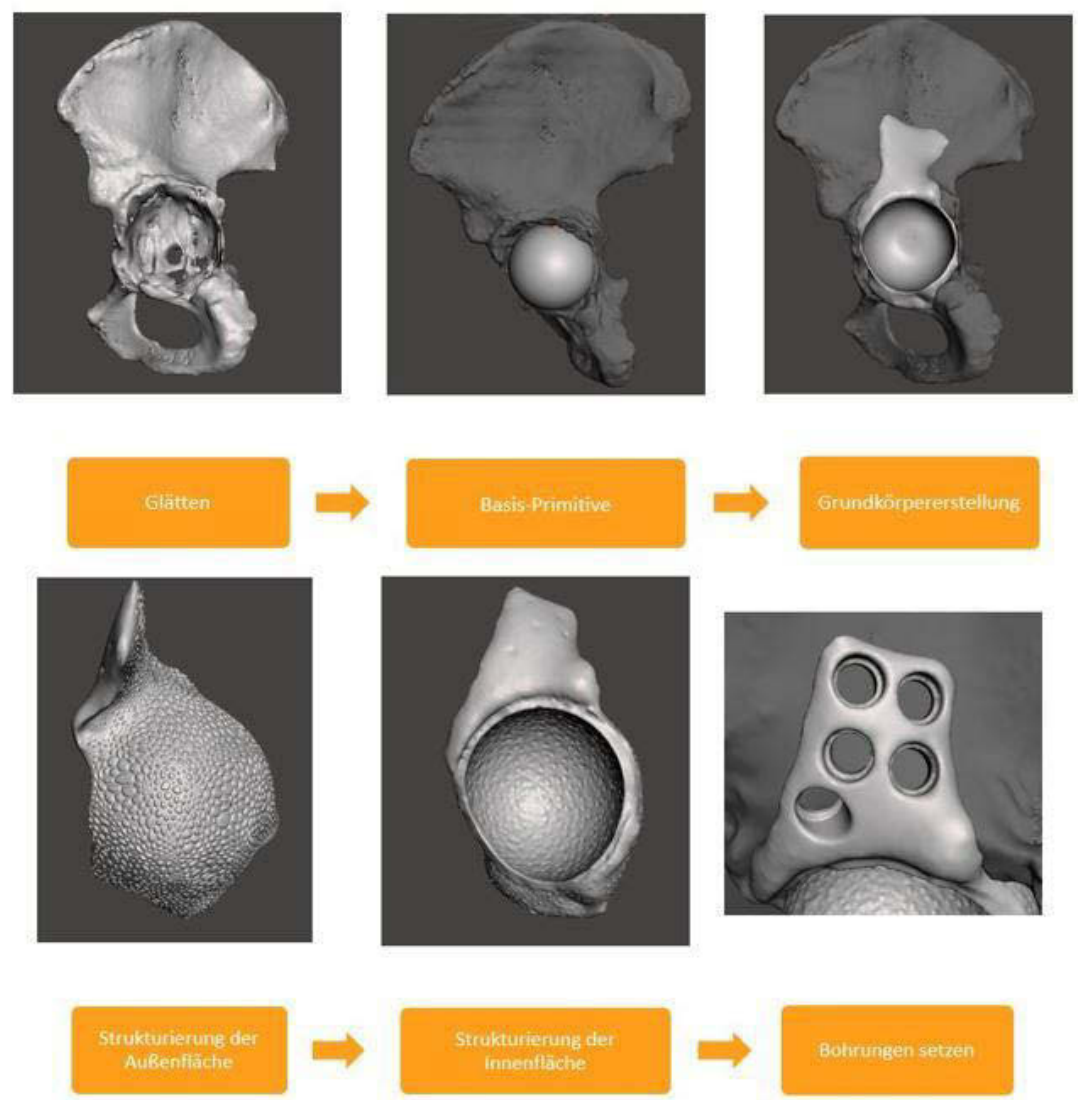

Figure 1: Ablauf der Rekonstruktion 


\section{DE GRUYTER}

sodass die Fixierung noch verstärkt wird. Dabei ist darauf zu achten, dass die Verschraubungen in den noch gesunden Bereichen mit ausreichend hoher Knochendichte gesetzt werden.

Im Anschluss an die Konstruktion wird das individualisierte Hüftpfannen-Implantat mit dem selektiven Laserschmelzen Verfahren (SLM) aus nichtrostenden 17-4 PH Stahl hergestellt.

\section{Ergebnisse}

\subsection{Medizinprodukteverordnung 2017/745/EG}

Das Einführen der neuen Medizinprodukteverordnung (MDR) soll zu einer stärkeren Überwachung der In-Verkehr gebrachten Medizinprodukte und deren Hersteller führen. Die Durchführung unangemeldeter Audits von den benannten Stellen ist eine neue Regelung, die dies bewirken soll. Außerdem werden die Anforderungen an benannten Stellen erhöht. Jedoch bleibt der Aspekt, dass diese Stellen weiterhin durch die Hersteller selbst finanziert werden und auch frei wählbar sind. Das bedeutet, dass auch nach einem vorherigen Ablehnen der Zulassung für das Produkt, die Hersteller das Verfahren des In-Verkehr Bringens erneut durchführen können.

Einige der jetzigen Klasse 2B Produkte fallen aufgrund einer neuen Klassifizierung nun in die Klasse 3 und müssen dadurch ein schärferes Konformitätsbewertungsverfahren durchlaufen, um das CE-Zeichen auf dem Produkt anbringen zu dürfen. Dies birgt vor allem einen sehr hohen Aufwand für bereits in Verkehr gebrachte Produkte. Zusätzlich tritt bei den Klasse 3 Produkten noch das sogenannte Scrutiny-Verfahren in Kraft. Bei dem gibt ein Expertengremium ihre Entscheidung der Akzeptanz oder Ablehnung der benannten Stelle preis. Ziel ist es, die benannte Stelle bei „Hochrisiko-Produkten“ zu unterstützen.

Für eine sichere und ausgeprägtere Dokumentation und eine höheren Transparenz soll die neue EUDAMED Datenbank und der UDI-Code eingeführt werden. In der Datenbank sollen alle Medizinprodukte erfasst werden, sodass jedes Produkt eindeutig identifizierbar ist und jegliche schwerwiegenden Ereignisse dokumentiert sind. Der UDICode muss auf jedem Medizinprodukt angebracht sein, um so jedes Produkt zweifellos zu identifizieren. [3, 4]

\subsection{Fertigungstechnik}

In den Figure 2 ist die individualisierte Endoprothese speziell für die Patientin zu sehen.

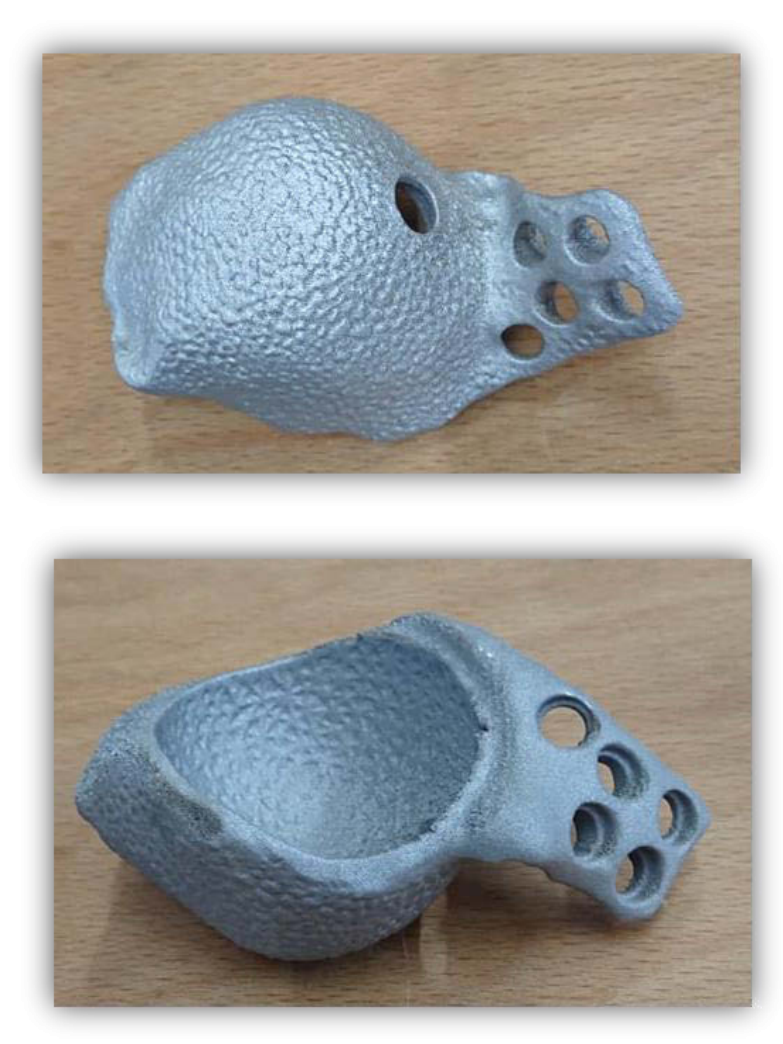

Figure 2: Individual Hüftpfannen-Endoprothese

\section{Diskussion}

\subsection{Medizinprodukteverordnung 2017/745/EG}

Im Gegensatz zur MDD gibt es bei der MDR nur kleine Änderungen, die für die personalisierte Endoprothetik von Relevanz sind, da für Sonderanfertigungen kein CE-Zeichen benötigt wird. Die Dokumentation und Rückführbarkeit soll erhöht werden. Daher werden mehr Daten erfasst, wie beispielsweise die Fertigungsstätten und alle eingehaltenen sowie nicht eingehaltenen Sicherheits- und Leistungsanforderungen mit Begründungen. Diese 
Dokumente müssen anstelle von fünf Jahren nun 15 Jahre aufbewahrt werden. Der Hersteller ist auch verpflichtet das Produkt nach der Implantation weiter zu verfolgen und bei schwerwiegenden Ereignissen, diese über die EUDAMED Datenbank zu melden. [3, 4]

\subsection{Fertigungstechnik}

Das Material 17-4PH ist nicht biokompatibel und somit nicht für die Implantation geeignet. Somit kann das gefertigte Implantat lediglich als Anschauungsmodell verwendet werden. Mithilfe des SLM Verfahrens ist es aber möglich, auch eine Endoprothese aus einer Titanlegierung wie TiAl6v4 herstellen zu können.

Die Oberflächenbeschaffenheit des Implantates ist in diesem Fall nicht geeignet für die Implantation in den Patienten. Durch die Limitierung des Programmes Meshmixer ist keine optimale Oberflächenrauheit möglich. Somit stellt dies nur ein Beispiel dar.

\section{Fazit}

Dieses Projekt hat den Prozess von den CT-Daten bis hin zum Prototypen eines Hüftpfannenimplantates durchlaufen. Lediglich die Beurteilung und Anpassung durch den Arzt und das Einsetzen sind bei einem vollständigen Ablauf weiterhin auszuführen.
Die Medizinprodukteverordnung spielt für die personalisierten Medizinprodukte eine untergeordnete Rolle. Lediglich die Dokumentation bei der Herstellung und die Überwachung von schwerwiegenden Ereignissen haben sich erhöht.

\section{Author Statement}

Research funding: The author state no funding involved. Conflict of interest: Authors state no conflict of interest. Informed consent: Informed consent has been obtained from all individuals included in this study. Ethical approval: The research related to human use complies with all the relevant national regulations, institutional policies and was performed in accordance with the tenets of the Helsinki Declaration, and has been approved by the authors' institutional review board or equivalent committee.

\section{References}

[1] Deutsche Gesellschaft für Orthopädie und orthopädische Chirurgie. Endoprothesenregister Deutschland (EPRD) Jahresbericht 2017. 2018.

[2] Paprosky W, Perona P, Lawrence J. Acetabular defect classification and surgical reconstruction in revision arthroplasty - A 6-year follow-up evaluation. 9. ed. The Journal of Arthroplasty. 1994.

[3] Europäisches Parlament und der Rat. 2017/745/EG Verordnung über Medizinprodukte. 2017.

[4] Rat der Europäischen Gemeinschaften. Richtlinie 93/42/EWG des Rates. 1993. 